\title{
Topology of superconductors beyond mean-field theory
}

\author{
Matthew F. Lapa $\odot^{*}$ \\ Kadanoff Center for Theoretical Physics, University of Chicago, Chicago, Illinois 60637, USA
}

(Received 24 March 2020; revised 26 June 2020; accepted 6 August 2020; published 26 August 2020)

\begin{abstract}
The study of topological superconductivity is largely based on the analysis of mean-field Hamiltonians that violate particle number conservation and have only short-range interactions. Although this approach has been very successful, it is not clear that it captures the topological properties of real superconductors, which are described by number-conserving Hamiltonians with long-range interactions. To address this issue, we study topological superconductivity directly in the number-conserving setting. We focus on a diagnostic for topological superconductivity that compares the fermion parity $\mathcal{P}$ of the ground state of a system in a ring geometry and in the presence of zero versus $\Phi_{\mathrm{sc}}=\frac{h}{2 e} \equiv \pi$ flux of an external magnetic field. A version of this diagnostic exists in any dimension and provides a $\mathbb{Z}_{2}$-invariant $v=\mathcal{P}_{0} \mathcal{P}_{\pi}$ for topological superconductivity. In this paper, we prove that the mean-field approximation correctly predicts the value of $v$ for a large family of number-conserving models of spinless superconductors. Our result applies directly to the cases of greatest physical interest, including $p$-wave and $p_{x}+i p_{y}$ superconductors in one and two dimensions, and gives strong evidence for the validity of the mean-field approximation in the study of (at least some aspects of) topological superconductivity.
\end{abstract}

DOI: 10.1103/PhysRevResearch.2.033309

\section{INTRODUCTION}

Topological superconductors (TSCs) [1-5] are of great interest both from a theoretical point of view and for their possible applications to quantum computation. However, most theoretical studies of TSCs rely on simple mean-field Hamiltonians that violate particle number conservation, and over the past few years several authors have expressed concerns about this approach [6-10]. In addition, on the experimental side, the interpretation of transport measurements designed to search for Majorana fermions is not yet clear [11-15]. To gain a better understanding of these issues, in Ref. [16] we initiated a rigorous study of TSCs in the more realistic number-conserving setting.

A key theoretical concern highlighted in Ref. [16] is the following. In the number-conserving setting, one must include long-range interactions to accurately describe real charged superconductors (e.g., to avoid the Goldstone theorem of Ref. [17]). However, it is not clear that the topological properties of number-conserving superconductor models with longrange interactions can be correctly captured by mean-field models that violate particle number conservation and have only short-range interactions.

In this paper, we address this concern by studying the topological phases of superconductors beyond mean-field theory. Specifically, we study a diagnostic for topological supercon-

\footnotetext{
*mlapa@uchicago.edu

Published by the American Physical Society under the terms of the Creative Commons Attribution 4.0 International license. Further distribution of this work must maintain attribution to the author(s) and the published article's title, journal citation, and DOI.
}

ductivity that compares the fermion parity $\mathcal{P}$ of the ground state [18] of a system in a ring geometry and in the presence of zero versus $\pi$ flux of an external magnetic field $[1,8,19]$. Here, $\pi$ flux is equivalent to one superconducting flux quantum $\Phi_{\mathrm{sc}}=\frac{h}{2 e}$ in units with $\hbar=e=1$. A version of this diagnostic exists in any spatial dimension and provides a $\mathbb{Z}_{2}$-invariant

$$
\nu=\mathcal{P}_{0} \mathcal{P}_{\pi}
$$

for topological superconductivity. The nontrivial phase corresponds to $v=-1$, where a "fermion parity switch" occurs between zero and $\pi$ flux. More physically, for mean-field models the value $v=-1$ indicates that gapless Majorana excitations appear at the boundaries of a finite system. The invariant $v$ is only well defined if the Hamiltonian at zero and $\pi$ flux possesses a finite parity gap, i.e., a finite energy gap between the ground state and the lowest energy state with opposite parity. Note also that $v$ only makes sense in the thermodynamic limit if the parity gaps remain finite in that limit.

We study the invariant $v$ in a general family of numberconserving pairing models of spinless fermions in $D$ spatial dimensions. We also restrict our attention to translationinvariant models, which allows us to apply the flux via a change in the boundary conditions. These pairing models are similar to the reduced BCS model [20] and to Richardson-type models [21,22], and they form a convenient starting point for the study of superconductivity in the number-conserving setting. In special cases, exactly solvable versions of these models have already been used to obtain detailed results on $p$ wave superconductors in $D=1$ and $p_{x}+i p_{y}$ superconductors (and more exotic cases) in $D=2[8,19,23-26]$. In addition, Ref. [19] proved that $v=-1$ in an exactly solvable model in $D=1[27]$. 
In this paper, we prove that for gapped pairing models $v$ satisfies the relation

$$
v=\prod_{\mathbf{k} \in \mathcal{K}_{0}} s_{\mathbf{k}},
$$

where $s_{\mathbf{k}}=\operatorname{sgn}\left(\epsilon_{\mathbf{k}}\right), \epsilon_{\mathbf{k}}$ is the single-particle energy dispersion in the pairing model, and $\mathcal{K}_{0}$ is the set of time-reversalinvariant wave vectors in the first Brillouin zone (these satisfy $\mathbf{k}=-\mathbf{k}+\mathbf{G}$ for some reciprocal lattice vector $\mathbf{G})$. This is exactly the result one would obtain for $v$ by studying the pairing model using a BCS-type mean-field approximation, which reduces the pairing model to a quadratic mean-field model. In addition, the product $\prod_{\mathbf{k} \in \mathcal{K}_{0}} s_{\mathbf{k}}$ is known to be a $\mathbb{Z}_{2}$ topological invariant for mean-field models of spinless superconductors in $D=1$ and $2[1,28]$. Therefore, our result proves that the mean-field approximation correctly predicts the value of $v$ for these gapped pairing models and, by the definition of $v$, for any model that is adiabatically connected to a gapped pairing model. For the precise statements of these results, see Theorem 1 and Corollary 1 below.

Previous studies of TSCs with number conservation have mostly focused on one-dimensional systems [8,16,19,29-42]. Important exceptions include Refs. [23-26], which considered exactly solvable pairing models in $D=2$, and Refs. $[9,10]$, which considered the braiding statistics of vortices in $D=2$. Our rigorous results for all $D \geqslant 1$ should nicely complement these previous studies and serve as a useful guide for future work on this topic.

This paper is organized as follows. In Sec. II, we introduce the family of number-conserving pairing models that we study in this paper. In Sec. III, we present our main results, and we also state and prove a lemma that is used in the proof of our main results. In Sec. IV, we present the proof of our main result (namely, Theorem 1 from Sec. III). Section V presents our conclusions. Finally, the Appendix contains an introduction to the reflection positivity property that is used our proof of Lemma 1 in Sec. III.

\section{NUMBER-CONSERVING PAIRING MODELS OF SPINLESS FERMIONS}

In this section, we introduce the pairing models that we study in this paper. The degrees of freedom in these models are spinless fermions $c_{\mathbf{x}}$ on the sites $\mathbf{x}$ of a Bravais lattice $\Lambda$ in $D$ spatial dimensions $(D \geqslant 1)$, and these operators obey standard anticommutation relations $\left\{c_{\mathbf{x}}, c_{\mathbf{y}}\right\}=0$ and $\left\{c_{\mathbf{x}}, c_{\mathbf{y}}^{\dagger}\right\}=\delta_{\mathbf{x y}}$. To avoid unnecessary complications, we also assume that the number of unit cells in the lattice in each coordinate direction is an even number.

We consider translation-invariant models with two different boundary conditions. In the first case, we consider periodic boundary conditions in all coordinate directions, corresponding to the absence of any magnetic flux. In the second case, we consider antiperiodic boundary conditions in a single coordinate direction, and periodic boundary conditions in the remaining $D-1$ directions. This corresponds to the presence of $\Phi_{\mathrm{sc}}=\frac{h}{2 e} \equiv \pi$ flux through a single hole in the $D$-dimensional torus on which our system lives. In $D=1$, the second case just corresponds to standard antiperiodic boundary conditions. In each case, the boundary conditions determine a set $\mathcal{K}$ of allowed wave vectors in the first Brillouin zone (BZ). For each allowed wave vector $\mathbf{k}$, we can define a fermion operator in reciprocal space via Fourier transform, $c_{\mathbf{k}}=|\Lambda|^{-1 / 2} \sum_{\mathbf{x}} c_{\mathbf{x}} e^{-i \mathbf{k} \cdot \mathbf{x}}$, where $|\Lambda|$ is the number of unit cells in the lattice.

We can always decompose $\mathcal{K}$ as $\mathcal{K}=\mathcal{K}_{0} \cup \mathcal{K}_{+} \cup \mathcal{K}_{-}$, where the three factors here are as follows. The first set $\mathcal{K}_{0}$ consists of all time-reversal-invariant wave vectors in the first BZ. These wave vectors satisfy $\mathbf{k}=-\mathbf{k}+\mathbf{G}$ for some reciprocal lattice vector $\mathbf{G}$. The remaining factor $\mathcal{K}_{+} \cup \mathcal{K}_{-}$ denotes any decomposition of the remaining wave vectors into two sets in such a way that, if $\mathbf{k} \in \mathcal{K}_{+}$, then $-\mathbf{k} \in \mathcal{K}_{-}$. The significance of the set $\mathcal{K}_{0}$ is that fermions at the wave vectors in this set do not participate in the pairing interaction in the Hamiltonians that we consider. A crucial point for the remainder of the paper is that in the case of antiperiodic boundary conditions we have $\mathcal{K}_{0}=\emptyset$; i.e., there are no time-reversal invariant wave vectors in the first BZ in the antiperiodic case.

It is helpful to illustrate our notation with an example. Consider a one-dimensional system in a ring geometry with $L$ sites and $L$ even (and with a lattice spacing equal to 1 ). Then in the periodic case we have $\mathcal{K}_{0}=\{0, \pi\}$ and $\mathcal{K}_{+}=$ $\left\{\frac{2 \pi}{L}, \frac{4 \pi}{L}, \ldots, \pi-\frac{2 \pi}{L}\right\}$, while in the antiperiodic case we have $\mathcal{K}_{0}=\emptyset$ and $\mathcal{K}_{+}=\left\{\frac{\pi}{L}, \frac{3 \pi}{L}, \ldots, \pi-\frac{\pi}{L}\right\}$.

The Hamiltonian for the pairing models that we consider, for either choice of boundary condition, takes the general form

$$
H=\sum_{\mathbf{k} \in \mathcal{K}} \epsilon_{\mathbf{k}} n_{\mathbf{k}}-\sum_{\mathbf{k}, \mathbf{k}^{\prime} \in \mathcal{K}_{+}} g_{\mathbf{k k}^{\prime}} c_{\mathbf{k}}^{\dagger} c_{-\mathbf{k}}^{\dagger} c_{-\mathbf{k}^{\prime}} c_{\mathbf{k}^{\prime}},
$$

where $n_{\mathbf{k}}=c_{\mathbf{k}}^{\dagger} c_{\mathbf{k}}, \epsilon_{\mathbf{k}}$ is a single-particle energy dispersion, and $g_{\mathbf{k k}^{\prime}}$ parametrizes the interaction between the pairs at $(\mathbf{k},-\mathbf{k})$ and $\left(\mathbf{k}^{\prime},-\mathbf{k}^{\prime}\right)$. Note that $H$ commutes with the total particle number operator $\mathcal{N}=\sum_{\mathbf{k}} c_{\mathbf{k}}^{\dagger} c_{\mathbf{k}}=\sum_{\mathbf{x}} c_{\mathbf{x}}^{\dagger} c_{\mathbf{x}}$. We also absorb any chemical potential term $-\mu \mathcal{N}$ into the definition of $\epsilon_{\mathbf{k}}$.

We make the following assumptions about $\epsilon_{\mathbf{k}}$ and $g_{\mathbf{k k}^{\prime}}$. First, we assume that $\epsilon_{\mathbf{k}}$ is an even function of $\mathbf{k}$ for $\mathbf{k} \notin \mathcal{K}_{0}$,

$$
\epsilon_{\mathbf{k}}=\epsilon_{-\mathbf{k}} \forall \mathbf{k} \in \mathcal{K}_{+} .
$$

Next, we assume that $g_{\mathbf{k k}^{\prime}}$ takes the factorized form

$$
g_{\mathbf{k} \mathbf{k}^{\prime}}=\bar{\eta}_{\mathbf{k}} \eta_{\mathbf{k}^{\prime}},
$$

where $\eta_{\mathbf{k}}$ is a complex function of $\mathbf{k}$ (the overline denotes complex conjugation), and we assume that $\eta_{\mathbf{k}} \neq 0$ for all $\mathbf{k} \in \mathcal{K}_{+}$. For specific examples of models of this form, which include the cases of $p$-wave superconductors in $D=1$ and $p_{x}+i p_{y}$ superconductors in $D=2$, we refer the reader to Refs. [8,19,23-26]. We note here that, unlike those references, we do not assume any fine-tuning of $\epsilon_{\mathbf{k}}$ or $\eta_{\mathbf{k}}$ that might lead to exact solvability.

One benefit of the factorization assumption (5) is that it implies that these pairing models also take a sensible form in real space. In this case, each individual sum $\sum_{\mathbf{k} \in \mathcal{K}_{+}} \eta_{\mathbf{k}} c_{-\mathbf{k}} c_{\mathbf{k}}$ in the pairing term can be Fourier transformed back to real space, and one finds that in real space the pairing term becomes a long-range pair hopping term.

Finally, let $\Delta_{0}$ and $\Delta_{\pi}$ be the parity gaps of the Hamiltonian with the two choices of boundary condition (which correspond to zero or $\pi$ flux). The invariant $\nu$ is only well defined if both of these gaps are nonzero. In the periodic case, 
we trivially find that $\Delta_{0}=0$ if $\epsilon_{\mathbf{k}}=0$ for any $\mathbf{k} \in \mathcal{K}_{0}$, and so in what follows we always assume that $\epsilon_{\mathbf{k}} \neq 0$ for all $\mathbf{k} \in \mathcal{K}_{0}$.

\section{MAIN RESULTS}

In this section, we present our main results (Theorem 1 and Corollary 1), and we also state and prove a lemma (Lemma 1) that is used in the proof of our main results. Our first result is a formula for $v$ in gapped pairing models.

Theorem 1. Let $H$ be a pairing Hamiltonian of the form (3) with nonzero parity gaps $\Delta_{0}$ and $\Delta_{\pi}$. Then for this Hamiltonian $v$ satisfies the relation

$$
v=\prod_{\mathbf{k} \in \mathcal{K}_{0}} s_{\mathbf{k}},
$$

where $s_{\mathbf{k}}=\operatorname{sgn}\left(\epsilon_{\mathbf{k}}\right)$ and $\mathcal{K}_{0}$ is the set of time-reversalinvariant wave vectors in the first Brillouin zone for the case of periodic boundary conditions.

By combining Theorem 1 with the definition of $\mathcal{P}_{0}$ and $\mathcal{P}_{\pi}$, we immediately obtain the following corollary.

Corollary 1. Let $H_{0}$ be a pairing Hamiltonian for which Theorem 1 applies, and let $H_{1}$ be any other translationinvariant Hamiltonian such that $H_{s}=(1-s) H_{0}+s H_{1}$ has nonzero parity gaps $\Delta_{0}(s)$ and $\Delta_{\pi}(s)$ for all $s \in[0,1]$. Then

$$
v_{1}=v_{0}=\prod_{\mathbf{k} \in \mathcal{K}_{0}} s_{\mathbf{k}},
$$

where $v_{0}$ and $v_{1}$ are the invariants for $H_{0}$ and $H_{1}$, respectively.

Theorem 1 shows that for gapped pairing models the invariant $v$ is equal to the value that one would predict using a BCStype mean-field approximation, namely the value $\prod_{\mathbf{k} \in \mathcal{K}_{0}} s_{\mathbf{k}}$. The product $\prod_{\mathbf{k} \in \mathcal{K}_{0}} s_{\mathbf{k}}$ is known to be a $\mathbb{Z}_{2}$ topological invariant for quadratic mean-field models of spinless superconductors, and in the mean-field context it was originally derived in Ref. [1] for the case of $D=1$ and Ref. [28] for $D=2$ (see also Refs. $[43,44]$ for $D>1$ ). Therefore, Theorem 1 and Corollary 1 prove that the mean-field approximation correctly predicts the value of $v$ for any translation-invariant model of spinless fermions that is adiabatically connected to a gapped pairing model. Note that for Corollary 1 we do not need to assume that $H_{1}$ is number conserving, but here we do assume that $H_{1}$ is translation invariant so that we can apply the $\pi$ flux via a change in the boundary conditions (i.e., by the choice of the set $\mathcal{K}$ of allowed wave vectors).

One possible application of Corollary 1 is to predict a topological superconducting phase in realistic Hamiltonians. For example, $H_{1}$ might be a Hamiltonian of the form

$$
H_{1}=\sum_{\mathbf{k} \in \mathcal{K}} \epsilon_{\mathbf{k}} n_{\mathbf{k}}+\sum_{\mathbf{x}, \mathbf{y} \in \Lambda} v_{\mathbf{x y}} n_{\mathbf{x}} n_{\mathbf{y}},
$$

where $v_{\mathbf{x y}}$ is a translation-invariant density-density interaction in real space $\left(n_{\mathbf{x}}=c_{\mathbf{x}}^{\dagger} c_{\mathbf{x}}\right)$. If $v_{\mathbf{x y}}$ favors a superconducting ground state with a finite-parity gap then, following the logic of the original BCS paper [20], it is possible that $H_{1}$ is adiabatically connected to a gapped pairing Hamiltonian of the form (3). In that case, we could then use Corollary 1 to predict whether $H_{1}$ supports a topological superconducting phase.
To prove Theorem 1, we first rewrite $H$ in the form $H=$ $\sum_{\mathbf{k} \in \mathcal{K}_{0}} \epsilon_{\mathbf{k}} n_{\mathbf{k}}+\tilde{H}$, where

$$
\tilde{H}=\sum_{\mathbf{k} \in \mathcal{K}_{+} \cup \mathcal{K}_{-}} \epsilon_{\mathbf{k}} n_{\mathbf{k}}-\sum_{\mathbf{k}, \mathbf{k}^{\prime} \in \mathcal{K}_{+}} g_{\mathbf{k} \mathbf{k}^{\prime}} c_{\mathbf{k}}^{\dagger} c_{-\mathbf{k}}^{\dagger} c_{-\mathbf{k}^{\prime}} c_{\mathbf{k}^{\prime}} .
$$

Note that $\tilde{H}$ only contains the fermions that participate in the pairing interaction. The proof of Theorem 1 relies on a lemma (Lemma 1) that concerns the ground state of $\tilde{H}$ acting within the space $\tilde{\mathcal{F}}$ consisting of those states annihilated by all the $c_{\mathbf{k}}$ with $\mathbf{k} \in \mathcal{K}_{0}$,

$$
\tilde{\mathcal{F}}=\left\{|\psi\rangle: c_{\mathbf{k}}|\psi\rangle=0 \forall \mathbf{k} \in \mathcal{K}_{0}\right\} .
$$

Let $\tilde{E}_{0}^{(M)}$ be the ground-state energy of $\tilde{H}$ in the $M$-particle sector of $\tilde{\mathcal{F}}$, and let $\left|\tilde{\psi}_{0}^{(M)}\right\rangle$ be the corresponding ground state (or a particular ground state if $\tilde{H}$ has a ground-state degeneracy in that sector). Note that $M$ must satisfy $0 \leqslant M \leqslant$ $|\Lambda|-\left|\mathcal{K}_{0}\right|$ (where $\left|\mathcal{K}_{0}\right|$ is the number of wave vectors in $\mathcal{K}_{0}$ ) since we are working within the space $\tilde{\mathcal{F}}$. Finally, let $M^{*}$, with $0 \leqslant M^{*} \leqslant|\Lambda|-\left|\mathcal{K}_{0}\right|$, be the (not necessarily unique) integer satisfying

$$
\tilde{E}_{0}^{(M)} \geqslant \tilde{E}_{0}^{\left(M^{*}\right)} \forall M \text { with } 0 \leqslant M \leqslant|\Lambda|-\left|\mathcal{K}_{0}\right| .
$$

Thus, $M^{*}$ (if it is unique) is the particle number in the ground state of $\tilde{H}$ acting in the space $\tilde{\mathcal{F}}$. The integer $M^{*}$ plays an important role in our proof below, and for this integer we have the following result.

Lemma 1. The integer $M^{*}$ can always be chosen to be even .

\section{A. Proof of Lemma 1}

We consider $\tilde{H}$ acting in the space $\tilde{\mathcal{F}}$. The proof is based on the fact that $\tilde{H}$ possesses the property of reflection positivity [45-47]. For the reader's benefit, we provide an introduction to this property in Appendix. In our case, the "reflection" one needs to consider is actually inversion in momentum space, $\mathbf{k} \rightarrow-\mathbf{k}$. However, we show below that $\tilde{H}$ can be mapped exactly to a Hamiltonian possessing Lieb's spin reflection positivity [46]. We can then immediately apply the results of Tian and Tang [48] on spinful pairing models to prove Lemma 1.

The Hamiltonian $\tilde{H}$ is written in terms of spinless fermions labeled by wave vectors $\mathbf{k}$ in both sets $\mathcal{K}_{+}$and $\mathcal{K}_{-}$. We now perform a change of variables to "spinful" fermions $c_{\mathbf{k}, \sigma}$, $\sigma \in\{\uparrow, \downarrow\}$, labeled by wave vectors in $\mathcal{K}_{+}$only. To define these new variables, we first decompose $\eta_{\mathbf{k}}$ into magnitude and phase parts as $\eta_{\mathbf{k}}=\left|\eta_{\mathbf{k}}\right| e^{i \theta_{\mathbf{k}}}$. Then, for any $\mathbf{k} \in \mathcal{K}_{+}$we define

$$
\begin{aligned}
c_{\mathbf{k}, \uparrow} & =c_{\mathbf{k}}, \\
c_{\mathbf{k}, \downarrow} & =e^{i \theta_{\mathbf{k}}} c_{-\mathbf{k}},
\end{aligned}
$$

and one can check that these operators obey standard anticommutation relations for spinful fermions. In terms of these new operators, $\tilde{H}$ can be written in the form

$$
\tilde{H}=\sum_{\mathbf{k} \in \mathcal{K}_{+}} \sum_{\sigma=\uparrow, \downarrow} \epsilon_{\mathbf{k}} n_{\mathbf{k}, \sigma}-\sum_{\mathbf{k}, \mathbf{k}^{\prime} \in \mathcal{K}_{+}}\left|\eta_{\mathbf{k}}\right|\left|\eta_{\mathbf{k}^{\prime}}\right| c_{\mathbf{k}, \uparrow}^{\dagger} c_{\mathbf{k}^{\prime}, \uparrow} c_{\mathbf{k}, \downarrow}^{\dagger} c_{\mathbf{k}^{\prime}, \downarrow},
$$

where $n_{\mathbf{k}, \sigma}=c_{\mathbf{k}, \sigma}^{\dagger} c_{\mathbf{k}, \sigma}$, and where we used $\epsilon_{\mathbf{k}}=\epsilon_{-\mathbf{k}}$ for $\mathbf{k} \in$ $\mathcal{K}_{+}$and also rearranged the order of the operators in the 
pairing term. With this change of variables $\tilde{H}$ now has the form of the pairing Hamiltonians for spinful fermions studied in Ref. [48] [see their Eq. (5)], and so it possesses Lieb's spin reflection positivity. We can then apply the results of Ref. [48] [specifically, their Eq. (26)] to conclude that

$$
\begin{aligned}
\tilde{E}_{0}^{(2 M+1)} & \geqslant \frac{1}{2}\left(\tilde{E}_{0}^{(2 M)}+\tilde{E}_{0}^{(2 M+2)}\right) \\
& \geqslant \min \left\{\tilde{E}_{0}^{(2 M)}, \tilde{E}_{0}^{(2 M+2)}\right\},
\end{aligned}
$$

which proves Lemma 1.

\section{PROOF OF THEOREM 1}

In this section, we present the proof of Theorem 1 . We first prove the theorem in the case of $D=1$. The proof for $D>1$ is conceptually identical to the $D=1$ case, but requires more cumbersome notation, and so we present the proof for $D>1$ after the proof for $D=1$.

\section{A. One-dimensional case}

For the case of $D=1$, we take $\Lambda$ to be a linear chain with $L$ sites and $L$ even (and with a lattice spacing equal to 1 ). With this choice, we have $\mathcal{K}_{0}=\{0, \pi\}$ for periodic boundary conditions (zero flux), while $\mathcal{K}_{0}=\emptyset$ for antiperiodic boundary conditions ( $\pi$ flux).

In the antiperiodic case, Lemma 1 and our assumption of a nonzero parity gap $\Delta_{\pi}$ immediately imply that $\mathcal{P}_{\pi}=1$. Therefore, it remains to compute $\mathcal{P}_{0}$. Our strategy for this is as follows. Let $E_{0}^{(N)}$ be the ground-state energy of $H$ in the $N$-particle sector. The first part of the proof consists of establishing (for any $N$ ) a lower bound on $E_{0}^{(N)}$ of the form $E_{0}^{(N)} \geqslant E_{0}^{\left(M^{* *}\right)}$, where $M^{* *}$ is a particular integer that we define below. Using our assumption of a nonzero parity gap $\Delta_{0}$, this implies that $\mathcal{P}_{0}=(-1)^{M^{* *}}$. Finally, we use Lemma 1 to prove that the integer $M^{* *}$ satisfies the relation $(-1)^{M^{* *}}=s_{0} s_{\pi}$, which completes the proof of Theorem 1 .

To start, let $\left|\psi_{0}^{(N)}\right\rangle$ be the ground state of $H$ in the $N$ particle sector (or one of the ground states if there is a degeneracy in that sector). We can always write $\left|\psi_{0}^{(N)}\right\rangle$ in the form

$$
\left|\psi_{0}^{(N)}\right\rangle=\sum_{b_{1}, b_{2}=0,1} a_{b_{1} b_{2}}\left(c_{0}^{\dagger}\right)^{b_{1}}\left(c_{\pi}^{\dagger}\right)^{b_{2}}\left|\psi_{b_{1} b_{2}}^{\left(N-b_{1}-b_{2}\right)}\right\rangle,
$$

where the four states $\left|\psi_{00}^{(N)}\right\rangle,\left|\psi_{10}^{(N-1)}\right\rangle,\left|\psi_{01}^{(N-1)}\right\rangle$, and $\left|\psi_{11}^{(N-2)}\right\rangle$ are all annihilated by $c_{0}$ and $c_{\pi}$ (i.e., they are states in $\tilde{\mathcal{F}}$ ), and where the coefficients $a_{b_{1} b_{2}}$ satisfy $\sum_{b_{1}, b_{2}=0,1}\left|a_{b_{1} b_{2}}\right|^{2}=1$. Note, however, that if $N=L-1$ then we must have $a_{00}=0$, and if $N=L$ then we must have $a_{00}=a_{10}=a_{01}=0$. On the other hand, if $N \leqslant L-2$, then all of $a_{b_{1} b_{2}}$ can be nonzero in general. In what follows, we consider the case where $N \leqslant L-2$ so that $a_{b_{1} b_{2}} \neq 0$ in general, but all of our results also hold for $N=L-1$ and $N=L$ and can be derived in the same way (but setting $a_{00}=0$ or $a_{00}=a_{10}=a_{01}=0$ from the start for the two cases of $N=L-1$ and $N=L$, respectively).
Using the fact that $\left[H, n_{0}\right]=\left[H, n_{\pi}\right]=0$, we find that $E_{0}^{(N)}=\left\langle\psi_{0}^{(N)}|H| \psi_{0}^{(N)}\right\rangle$ takes the form

$$
\begin{aligned}
E_{0}^{(N)}= & \left|a_{00}\right|^{2}\left\langle\psi_{00}^{(N)}|\tilde{H}| \psi_{00}^{(N)}\right\rangle \\
& +\left|a_{10}\right|^{2}\left(\epsilon_{0}+\left\langle\psi_{10}^{(N-1)}|\tilde{H}| \psi_{10}^{(N-1)}\right\rangle\right) \\
& +\left|a_{01}\right|^{2}\left(\epsilon_{\pi}+\left\langle\psi_{01}^{(N-1)}|\tilde{H}| \psi_{01}^{(N-1)}\right\rangle\right) \\
& +\left|a_{11}\right|^{2}\left(\epsilon_{0}+\epsilon_{\pi}+\left\langle\psi_{11}^{(N-2)}|\tilde{H}| \psi_{11}^{(N-2)}\right\rangle\right) .
\end{aligned}
$$

Next, using the variational theorem for the ground state of $\tilde{H}$ restricted to $\tilde{\mathcal{F}}$ (e.g., $\left\langle\psi_{00}^{(N)}|\tilde{H}| \psi_{00}^{(N)}\right\rangle \geqslant \tilde{E}_{0}^{(N)}$ ), the fact that $\tilde{E}_{0}^{(M)} \geqslant \tilde{E}_{0}^{\left(M^{*}\right)}$ for any $M \leqslant L-2$, and $\sum_{b_{1}, b_{2}=0,1}\left|a_{b_{1} b_{2}}\right|^{2}=1$, we find that

$$
E_{0}^{(N)} \geqslant \tilde{E}_{0}^{\left(M^{*}\right)}+\left(\left|a_{10}\right|^{2}+\left|a_{11}\right|^{2}\right) \epsilon_{0}+\left(\left|a_{01}\right|^{2}+\left|a_{11}\right|^{2}\right) \epsilon_{\pi} .
$$

To proceed further, we define $h_{0}=\left(1-s_{0}\right) / 2$ and $h_{\pi}=$ $\left(1-s_{\pi}\right) / 2$, and note that $h_{0} \in\{0,1\}$ and likewise for $h_{\pi}$. The inequality (17), combined with the constraint $\sum_{b_{1}, b_{2}=0,1}\left|a_{b_{1} b_{2}}\right|^{2}=1$, then implies that

$$
E_{0}^{(N)} \geqslant h_{0} \epsilon_{0}+h_{\pi} \epsilon_{\pi}+\tilde{E}_{0}^{\left(M^{*}\right)} .
$$

This inequality holds for any value of $N$, although in our derivation here we considered the case of $N \leqslant L-2$.

The next step of the proof is to study the trial state

$$
\left|\psi_{t}\right\rangle=\left(c_{0}^{\dagger}\right)^{h_{0}}\left(c_{\pi}^{\dagger}\right)^{h_{\pi}}\left|\tilde{\psi}_{0}^{\left(M^{*}\right)}\right\rangle
$$

where $\left|\tilde{\psi}_{0}^{\left(M^{*}\right)}\right\rangle$ is the ground state of $\tilde{H}$ in the $M^{*}$-particle sector of $\tilde{\mathcal{F}}$ (or one of the ground states if $\tilde{H}$ has a degeneracy in that sector). This trial state has a particle number equal to

$$
M^{* *}=M^{*}+h_{0}+h_{\pi} .
$$

In addition, the energy of this trial state is

$$
E_{t}=\left\langle\psi_{t}|H| \psi_{t}\right\rangle=h_{0} \epsilon_{0}+h_{\pi} \epsilon_{\pi}+\tilde{E}_{0}^{\left(M^{*}\right)} .
$$

From this, we can see that the inequality (18) can be rewritten in the form

$$
E_{0}^{(N)} \geqslant E_{t} \forall N
$$

On the other hand, using the variational theorem for $H$ in the $M^{* *}$-particle sector, we have the upper bound $E_{0}^{\left(M^{* *}\right)} \leqslant E_{t}$. Combining this upper bound with the lower bound (22) yields an equality for the ground-state energy in the $M^{* *}$-particle sector, $E_{0}^{\left(M^{* *}\right)}=E_{t}$. Then our previous inequality (22) can be rewritten as

$$
E_{0}^{(N)} \geqslant E_{0}^{\left(M^{* *}\right)} \forall N .
$$

This inequality shows that the ground-state energy in any sector of fixed particle number is greater than or equal to the ground-state energy in the $M^{* *}$-particle sector. By combining this inequality with our assumption of a nonzero parity gap $\Delta_{0}$, we find that $\mathcal{P}_{0}=(-1)^{M^{* *}}$.

Finally, we come to the crucial point. Using Lemma 1, which implies that we can take $M^{*}$ to be even, we find that

$$
(-1)^{M^{* *}}=(-1)^{h_{0}+h_{\pi}}=s_{0} s_{\pi} .
$$

This completes the proof of Theorem 1 for the case of $D=1$. 


\section{B. Higher dimensions}

In this section, we prove Theorem 1 in any spatial dimension $D \geqslant 1$. The logic of the proof is exactly the same as in the $D=1$ case from the previous subsection. To start, since $\mathcal{K}_{0}=\emptyset$ in the antiperiodic case ( $\pi$ flux), our assumption of a nonzero parity gap $\Delta_{\pi}$ again implies that $\mathcal{P}_{\pi}=1$. So all that remains is to again calculate $\mathcal{P}_{0}$.

We now introduce some notation that will streamline the calculation of $\mathcal{P}_{0}$ in this higher-dimensional case. Let $H$ be the pairing Hamiltonian with periodic boundary conditions, let $E_{0}^{(N)}$ be the ground-state energy of $H$ in the $N$-particle sector, and let $\left|\psi_{0}^{(N)}\right\rangle$ be the ground state of $H$ in the $N$-particle sector (or one of the ground states if $H$ has a degeneracy in that sector). In addition, let $\mathbf{k}_{j}$, for $j \in\left\{1, \ldots,\left|\mathcal{K}_{0}\right|\right\}$, be the wave vectors in the set $\mathcal{K}_{0}$, and let $s_{j}=\operatorname{sgn}\left(\epsilon_{\mathbf{k}_{j}}\right)$ and $h_{j}=\left(1-s_{j}\right) / 2$ (and recall that we assume that $\epsilon_{\mathbf{k}} \neq$ 0 for all $\mathbf{k} \in \mathcal{K}_{0}$ to avoid a trivial vanishing of the parity gap $\left.\Delta_{0}\right)$.

To start, we note that we can again write $\left|\psi_{0}^{(N)}\right\rangle$ as a linear combination of states with different occupations of the fermions labeled by wave vectors in $\mathcal{K}_{0}$. In particular, we can write

$$
\left|\psi_{0}^{(N)}\right\rangle=\sum_{b_{1}, \ldots, b_{\left|\mathcal{K}_{0}\right|}=0,1} a_{b_{1} \ldots b_{\left|\mathcal{K}_{0}\right|}}\left(c_{\mathbf{k}_{1}}^{\dagger}\right)^{b_{1}} \ldots\left(c_{\mathbf{k}_{\left|\mathcal{K}_{0}\right|}^{\dagger}}^{\dagger}\right)^{b_{\left|\mathcal{K}_{0}\right|} \mid}\left|\psi_{b_{1} \ldots b_{\mid} \mathcal{K}_{0} \mid}^{\left(N-\sum_{j=1}^{\left|\mathcal{K}_{0}\right|} b_{j}\right)}\right\rangle
$$

where the $2^{\left|\mathcal{K}_{0}\right|}$ states $\left|\psi_{b_{1} \cdots b_{\mid} \mathcal{K}_{0} \mid}^{\left(N-\sum_{j=1}^{\left|\mathcal{K}_{0}\right|} b_{j}\right)}\right\rangle$ are annihilated by $c_{\mathbf{k}_{j}}$ for all $j$ (i.e., these states lie in the space $\tilde{\mathcal{F}}$ ), and where the coefficients $a_{b_{1} \cdots b_{\left|\mathcal{K}_{0}\right|}}$ satisfy

$$
\sum_{b_{1}, \ldots, b_{\left|\mathcal{K}_{0}\right|}=0,1}\left|a_{b_{1} \ldots b_{\left|\mathcal{K}_{0}\right|}}\right|^{2}=1 .
$$

As in the one-dimensional case, some of the coefficients $a_{b_{1} \cdots b_{\left|\mathcal{K}_{0}\right|}}$ may be zero depending on the specific value of $N$, and all of these coefficients can be nonzero for $N \leqslant|\Lambda|-$ $\left|\mathcal{K}_{0}\right|$.

As in the $D=1$ case, we can use this expression for $\left|\psi_{0}^{(N)}\right\rangle$ and the fact that $\left[H, n_{\mathbf{k}_{j}}\right]=0$ for all $j$ to obtain the lower bound

$$
E_{0}^{(N)} \geqslant \sum_{j=1}^{\left|\mathcal{K}_{0}\right|} h_{j} \epsilon_{\mathbf{k}_{j}}+\tilde{E}_{0}^{\left(M^{*}\right)} .
$$

We then define the trial state

$$
\left|\psi_{t}\right\rangle=\left(c_{\mathbf{k}_{1}}^{\dagger}\right)^{h_{1}} \ldots\left(c_{\mathbf{k}_{\left|\mathcal{K}_{0}\right|}^{\dagger}}^{\dagger}\right)^{h_{\left|\mathcal{K}_{0}\right|} \mid}\left|\tilde{\psi}_{0}^{\left(M^{*}\right)}\right\rangle,
$$

where $\left|\tilde{\psi}_{0}^{\left(M^{*}\right)}\right\rangle$ is the ground state of $\tilde{H}$ in the $M^{*}$-particle sector of $\tilde{\mathcal{F}}$ (or one of the ground states if $\tilde{H}$ has a groundstate degeneracy in that sector). This trial state has a particle number equal to $M^{* *}$, where now

$$
M^{* *}=M^{*}+\sum_{j=1}^{\left|\mathcal{K}_{0}\right|} h_{j},
$$

and the energy of this state is given by

$$
E_{t}=\left\langle\psi_{t}|H| \psi_{t}\right\rangle=\sum_{j=1}^{\left|\mathcal{K}_{0}\right|} h_{j} \epsilon_{\mathbf{k}_{j}}+\tilde{E}_{0}^{\left(M^{*}\right)} .
$$

Using the same variational arguments from the previous subsection, we again find that $E_{0}^{\left(M^{* *}\right)}=E_{t}$ and that $E_{0}^{(N)} \geqslant E_{0}^{\left(M^{* *}\right)}$ for all $N$. Finally, we can again apply our assumption of a nonzero parity gap $\Delta_{0}$, and the result of Lemma 1 , to find that

$$
\mathcal{P}_{0}=(-1)^{M^{* *}}=(-1)^{\sum_{j=1}^{\left|\mathcal{K}_{0}\right|} h_{j}}=\prod_{j=1}^{\left|\mathcal{K}_{0}\right|} s_{j}=\prod_{\mathbf{k} \in \mathcal{K}_{0}} s_{\mathbf{k}} .
$$

This completes the proof of Theorem 1 for a general spatial dimension $D \geqslant 1$.

\section{DISCUSSION AND CONCLUSION}

We have proven that the mean-field approximation correctly predicts the value of the $\mathbb{Z}_{2}$ topological invariant $v=$ $\mathcal{P}_{0} \mathcal{P}_{\pi}$ for any translation-invariant Hamiltonian that is adiabatically connected to a gapped pairing model of the form (3). We emphasize that this is a large family of models that is likely to contain many realistic models with a superconducting ground state. Our rigorous results give strong evidence that the mean-field approach is reliable, at least for the calculation of bulk topological invariants. As a topic for future work, we propose to search for evidence of Majorana-like excitations in pairing models with interfaces or boundaries, as our results strongly suggest that some kind of interesting gapless excitations should appear at the boundary between two pairing models with opposite values of $v$. Such a study would also have a direct impact on future experiments on TSCs, as the number-conserving pairing models are (presumably) a better description of the true experimental situation than the mean-field models.

\section{ACKNOWLEDGMENTS}

We thank M. Levin for many discussions on this topic and for giving many helpful comments on the first draft of this paper. We also acknowledge the support of the Kadanoff Center for Theoretical Physics at the University of Chicago. This work was supported by the Simons Collaboration on Ultra-Quantum Matter, which is a grant from the Simons Foundation (651440)

\section{APPENDIX: REFLECTION POSITIVITY OF THE PAIRING HAMILTONIAN $\tilde{H}$}

In this Appendix, we introduce reflection positivity in the setting relevant for our work, and then we show that the pairing Hamiltonian $\tilde{H}$ [Eq. (9) of the main text] is indeed reflection positive. An interested reader should then be able to consult Ref. [48] to see how this property is used to 
prove Eq. (14) of the main text. Although the term "reflection positivity" has slightly different meanings in different areas of physics (e.g., in quantum field theory, statistical mechanics, quantum mechanics, etc.), in our setting reflection positivity is simply the statement that the Hamiltonian of the system takes a certain special form. Some useful references for reflection positivity in this sense are Refs. [45,46] and Appendix 2 of Ref. [47]. The special form of a reflection-positive Hamiltonian is extremely useful because it allows for the derivation of various inequalities that can be used to prove many things about the model.

We first describe the general form of a reflection-positive Hamiltonian. We start with a Hilbert space $\mathcal{H}$. A reflectionpositive Hamiltonian acts on the tensor product space $\mathcal{H} \otimes \mathcal{H}$ and takes a specific form that we now describe. Let $A$ be a Hermitian operator on $\mathcal{H}$, let $\mathcal{J}$ be some index set, and let $B_{J}$, for all $J \in \mathcal{J}$, be a set of real operators on $\mathcal{H}$ (i.e., the matrix elements of the $B_{J}$ are real in a certain preferred basis for $\mathcal{H}$ ). In addition, let $g_{J} \geqslant 0$ be a set of non-negative real coefficients labeled by $J \in \mathcal{J}$, and assume that $\sum_{J \in \mathcal{J}} g_{J} B_{J} \otimes$ $B_{J}$ is symmetric as an operator on $\mathcal{H} \otimes \mathcal{H}$. In terms of these ingredients, a reflection-positive Hamiltonian $H_{\mathrm{RP}}$ acting on $\mathcal{H} \otimes \mathcal{H}$ takes the form

$$
H_{\mathrm{RP}}=A \otimes \mathbb{I}+\mathbb{I} \otimes A-\sum_{J} g_{J} B_{J} \otimes B_{J} .
$$

The minus sign on the term with the $B_{J}$ (and the nonnegativity of the $g_{J}$ ) is crucial for reflection positivity. When the two factors of $\mathcal{H}$ represent spin-up and spin-down degrees of freedom, a system with a Hamiltonian of the form $H_{\mathrm{RP}}$ is said to possess Lieb's spin reflection positivity [46].

We now show that the pairing Hamiltonian $\tilde{H}$ from Eq. (9) of the main text is indeed a Hamiltonian of the form (A1). We consider $\tilde{H}$ acting within the Fock space $\mathcal{F}_{\mathcal{K}_{+} \cup \mathcal{K}_{-}}$for the fermions labeled by wave vectors $\mathbf{k}$ in the set $\mathcal{K}_{+} \cup \mathcal{K}_{-}$[note that this Fock space is equivalent to the space $\tilde{\mathcal{F}}$ that we defined in Eq. (10) of the main text]. When written in terms of the spinful fermion operators $c_{\mathbf{k}, \sigma}$ that we introduced in the main text (with $\mathbf{k} \in \mathcal{K}_{+}$and $\sigma \in\{\uparrow, \downarrow\}$ ), $\tilde{H}$ takes the form

$$
\tilde{H}=\sum_{\mathbf{k} \in \mathcal{K}_{+}} \sum_{\sigma=\uparrow, \downarrow} \epsilon_{\mathbf{k}} n_{\mathbf{k}, \sigma}-\sum_{\mathbf{k}, \mathbf{k}^{\prime} \in \mathcal{K}_{+}}\left|\eta_{\mathbf{k}}\right|\left|\eta_{\mathbf{k}^{\prime}}\right| c_{\mathbf{k}, \uparrow}^{\dagger} c_{\mathbf{k}^{\prime}, \uparrow} c_{\mathbf{k}, \downarrow}^{\dagger} c_{\mathbf{k}^{\prime}, \downarrow} .
$$

To proceed, we define separate number operators for the spin-up and spin-down fermions, $\mathcal{N}_{\sigma}=\sum_{\mathbf{k} \in \mathcal{K}_{+}} n_{\mathbf{k}, \sigma}$. We then use these number operators to define new spinful fermion operators $C_{\mathbf{k}, \sigma}$ as follows. For spin-up, we set $C_{\mathbf{k}, \uparrow}$ equal to $c_{\mathbf{k}, \uparrow}$,

$$
C_{\mathbf{k}, \uparrow}=c_{\mathbf{k}, \uparrow} .
$$

On the other hand, for spin-down we define $C_{\mathbf{k}, \downarrow}$ via

$$
C_{\mathbf{k}, \downarrow}=(-1)^{\mathcal{N}_{\uparrow}} c_{\mathbf{k}, \downarrow} .
$$

With this definition, we still find that $\left\{C_{\mathbf{k}, \sigma}, C_{\mathbf{k}^{\prime}, \sigma}^{\dagger}\right\}=\delta_{\mathbf{k k}^{\prime}}$ and $\left\{C_{\mathbf{k}, \sigma}, C_{\mathbf{k}^{\prime}, \sigma}\right\}=0$ for operators with the same spin, but now we find that operators with opposite spins commute instead of anticommute, for example,

$$
\left[C_{\mathbf{k}, \uparrow}, C_{\mathbf{k}^{\prime}, \downarrow}\right]=\left[C_{\mathbf{k}, \uparrow}, C_{\mathbf{k}^{\prime}, \downarrow}^{\dagger}\right]=0 .
$$

On the other hand, $\tilde{H}$ takes the exact same form when written in terms of the new fermion operators,

$\tilde{H}=\sum_{\mathbf{k} \in \mathcal{K}_{+}} \sum_{\sigma=\uparrow, \downarrow} \epsilon_{\mathbf{k}} N_{\mathbf{k}, \sigma}-\sum_{\mathbf{k}, \mathbf{k}^{\prime} \in \mathcal{K}_{+}}\left|\eta_{\mathbf{k}}\right|\left|\eta_{\mathbf{k}^{\prime}}\right| C_{\mathbf{k}, \uparrow}^{\dagger} C_{\mathbf{k}^{\prime}, \uparrow} C_{\mathbf{k}, \downarrow}^{\dagger} C_{\mathbf{k}^{\prime}, \downarrow}$,

where we defined $N_{\mathbf{k}, \sigma}=C_{\mathbf{k}, \sigma}^{\dagger} C_{\mathbf{k}, \sigma}$.

We are now ready to show that $\tilde{H}$ is reflection positive. Since the spin-up fermions $C_{\mathbf{k}, \uparrow}$ commute with the spin-down fermions $C_{\mathbf{k}, \downarrow}$, we can now regard the Hamiltonian $\tilde{H}$ as acting on the tensor product space

$$
\mathcal{F}_{\mathcal{K}_{+}} \otimes \mathcal{F}_{\mathcal{K}_{+}},
$$

where $\mathcal{F}_{\mathcal{K}_{+}}$is the Fock space for a single set of fermions $C_{\mathbf{k}}$ labeled only by wave vectors $\mathbf{k}$ in $\mathcal{K}_{+}$(no additional spin index). We now see that $\tilde{H}$ can be written in the reflection positive form (A1), if we make the following identifications. First, the Hilbert space $\mathcal{H}$ is equal to the Fock space $\mathcal{F}_{\mathcal{K}_{+}}$for the fermions operators $C_{\mathbf{k}}$. Next, the Hermitian operator $A$ is given by

$$
A=\sum_{\mathbf{k} \in \mathcal{K}_{+}} \epsilon_{\mathbf{k}} N_{\mathbf{k}}
$$

where $N_{\mathbf{k}}=C_{\mathbf{k}}^{\dagger} C_{\mathbf{k}}$. Finally, the abstract index $J$ is identified with pairs $\left(\mathbf{k}, \mathbf{k}^{\prime}\right)$ of wave vectors in $\mathcal{K}_{+}, J=\left(\mathbf{k}, \mathbf{k}^{\prime}\right)$, and the operators $B_{J}$ and coefficients $g_{J}$ are given by

$$
B_{J}=B_{\left(\mathbf{k}, \mathbf{k}^{\prime}\right)}=C_{\mathbf{k}}^{\dagger} C_{\mathbf{k}^{\prime}}
$$

and

$$
g_{J}=g_{\left(\mathbf{k}, \mathbf{k}^{\prime}\right)}=\left|\eta_{\mathbf{k}}\right|\left|\eta_{\mathbf{k}^{\prime}}\right| .
$$

One can also check that the $B_{J}$ operators have real matrix elements in the occupation number basis for $\mathcal{F}_{\mathcal{K}_{+}}$in which all the number operators $N_{\mathbf{k}}$ are diagonal.

This completes our introduction to reflection positivity and our demonstration that the pairing Hamiltonian $\tilde{H}$ has this property. This information should allow any interested reader to consult Ref. [48] to see how reflection positivity is used to prove Eq. (14) of the main text.
[1] A. Y. Kitaev, Phys. Usp. 44, 131 (2001).

[2] N. Read and D. Green, Phys. Rev. B 61, 10267 (2000).

[3] Y. Tanaka, M. Sato, and N. Nagaosa, J. Phys. Soc. Jpn. 81, 011013 (2011).

[4] J. Alicea, Rep. Prog. Phys. 75, 076501 (2012).

[5] M. Sato and Y. Ando, Rep. Prog. Phys. 80, 076501 (2017).
[6] A. J. Leggett, http://people.physics.illinois.edu/Leggett/MZM. pdf.

[7] A. J. Leggett, https://www.youtube.com/playlist?list=PLBRgy tHojT9YMGLNaVjowfn829AZY1kGQ.

[8] G. Ortiz and E. Cobanera, Ann. Phys. 372, 357 (2016).

[9] Y. Lin and A. J. Leggett, arXiv:1708.02578.

[10] Y. Lin and A. J. Leggett, arXiv:1803.08003. 
[11] J. Chen, B. D. Woods, P. Yu, M. Hocevar, D. Car, S. R. Plissard, E. P. A. M. Bakkers, T. D. Stanescu, and S. M. Frolov, Phys. Rev. Lett. 123, 107703 (2019).

[12] B. D. Woods, J. Chen, S. M. Frolov, and T. D. Stanescu, Phys. Rev. B 100, 125407 (2019).

[13] W. Ji and X.-G. Wen, Phys. Rev. Lett. 120, 107002 (2018).

[14] Y. Huang, F. Setiawan, and J. D. Sau, Phys. Rev. B 97, 100501(R) (2018).

[15] M. Kayyalha, D. Xiao, R. Zhang, J. Shin, J. Jiang, F. Wang, Y.-F. Zhao, R. Xiao, L. Zhang, K. M. Fijalkowski, P. Mandal, M. Winnerlein, C. Gould, Q. Li, L. W. Molenkamp, M. H. W. Chan, N. Samarth, and C.-Z. Chang, Science 367, 64 (2020).

[16] M. F. Lapa and M. Levin, Phys. Rev. Lett. 124, 257002 (2020).

[17] M. B. Hastings, J. Stat. Mech.: Theory Exp. (2007) P05010.

[18] For a model with a conserved total particle number, by "ground state" we mean the lowest energy state over all possible particle number sectors.

[19] G. Ortiz, J. Dukelsky, E. Cobanera, C. Esebbag, and C. Beenakker, Phys. Rev. Lett. 113, 267002 (2014).

[20] J. Bardeen, L. N. Cooper, and J. R. Schrieffer, Phys. Rev. 108, 1175 (1957).

[21] R. W. Richardson and N. Sherman, Nucl. Phys. 52, 221 (1964).

[22] R. W. Richardson, J. Math. Phys. 6, 1034 (1965).

[23] M. Ibañez, J. Links, G. Sierra, and S.-Y. Zhao, Phys. Rev. B 79, 180501(R) (2009).

[24] C. Dunning, M. Ibanez, J. Links, G. Sierra, and S.-Y. Zhao, J. Stat. Mech.: Theory Exp. (2010) P08025.

[25] S. M. A. Rombouts, J. Dukelsky, and G. Ortiz, Phys. Rev. B 82, 224510 (2010).

[26] L. Lepori and M. Roncaglia, Phys. Rev. B 98, 144504 (2018).

[27] In this paper, we define $v$ using the parity of the lowest energy state of a given Hamiltonian over all possible particle number sectors. This should be contrasted with the approach in Ref. [19], where the authors considered a fixed $N$ and checked whether the sign of $E_{0}^{(N)}-\frac{1}{2}\left(E_{0}^{(N-1)}+E_{0}^{(N+1)}\right)$ was different for the two choices of boundary condition (here, $E_{0}^{(N)}$ is the ground-state energy in the $N$-particle sector).

[28] M. Sato, Phys. Rev. B 81, 220504(R) (2010).

[29] M. Cheng and H.-H. Tu, Phys. Rev. B 84, 094503 (2011).

[30] L. Fidkowski, R. M. Lutchyn, C. Nayak, and M. P. A. Fisher, Phys. Rev. B 84, 195436 (2011).

[31] J. D. Sau, B. I. Halperin, K. Flensberg, and S. Das Sarma, Phys. Rev. B 84, 144509 (2011).

[32] A. M. Tsvelik, arXiv:1106.2996.

[33] M. Cheng and R. Lutchyn, Phys. Rev. B 92, 134516 (2015).

[34] C. L. Kane, A. Stern, and B. I. Halperin, Phys. Rev. X 7, 031009 (2017).

[35] C. Knapp, J. I. Väyrynen, and R. M. Lutchyn, Phys. Rev. B 101, 125108 (2020).

[36] F. Iemini, L. Mazza, D. Rossini, R. Fazio, and S. Diehl, Phys. Rev. Lett. 115, 156402 (2015).

[37] N. Lang and H. P. Büchler, Phys. Rev. B 92, 041118(R) (2015).

[38] Z. Wang, Y. Xu, H. Pu, and K. R. A. Hazzard, Phys. Rev. B 96, 115110 (2017).

[39] C. V. Kraus, M. Dalmonte, M. A. Baranov, A. M. Läuchli, and P. Zoller, Phys. Rev. Lett. 111, 173004 (2013).

[40] C. Chen, W. Yan, C. S. Ting, Y. Chen, and F. J. Burnell, Phys. Rev. B 98, 161106(R) (2018).

[41] J. Ruhman and E. Altman, Phys. Rev. B 96, 085133 (2017).

[42] X. Yin, T.-L. Ho, and X. Cui, New J. Phys. 21, 013004 (2019).

[43] M. Sato, Phys. Rev. B 79, 214526 (2009).

[44] L. Fu and E. Berg, Phys. Rev. Lett. 105, 097001 (2010).

[45] T. Kennedy, E. H. Lieb, and B. S. Shastry, J. Stat. Phys. 53, 1019 (1988).

[46] E. H. Lieb, Phys. Rev. Lett. 62, 1201 (1989).

[47] E. H. Lieb and B. Nachtergaele, Phys. Rev. B 51, 4777 (1995).

[48] G.-S. Tian and L.-H. Tang, Phys. Rev. B 58, 12333 (1998). 